\title{
Long term Fermi-LAT and multi-band observations of blazar 3C 279
}

\section{Masaaki Hayashida}

Kavli Institute for Particle Astrophysics and Cosmology, SLAC National Accelerator Laboratory, Stanford University, CA, 94025, USA

E-mail: mahayadslac.stanford.edu

\section{Greg Madejski}

Kavli Institute for Particle Astrophysics and Cosmology, SLAC National Accelerator Laboratory,

Stanford University, CA, 94025, USA

E-mail: madejski@slac.stanford.edu

\section{for the Fermi-LAT Collaboration and members of the 3C 279 multi-band campaign}

The flat Spectrum Radio Quasar 3C 279 was one of the brightest gamma-ray blazars in the sky at the time of the discovery with EGRET. Since the successful launch of the Fermi Gamma-ray Space telescope in 2008, we have organized extensive multi-band campaign on 3C 279 from radio to gamma-ray bands as well as optical polarimetric observations. The uninterrupted monitoring in the gamma-ray band by Fermi-LAT together with the multi-band data provide us with new insights into the relativistic jet of the blazar. Here, we present the results from the observational campaign including the discovery of a gamma-ray flare event associated with a dramatic change of the optical polarization - as well as a discovery of an "orphan" X-ray flare, unassociated with prominent outbursts in other bands.

25th Texas Symposium on Relativistic Astrophysics - TEXAS 2010

December 06-10, 2010

Heidelberg, Germany 


\section{Introduction}

Active galactic nuclei (AGNs) with strong relativistic jets which are pointed close to our line of sight are classified as "blazars". Blazars are in fact most numerous bright and generally variable extragalactic $\gamma$-ray sources detected by Fermi, as expected from the EGRET days [2]. The radiative processes operating in those jets are most likely the synchrotron process responsible for the radiothrough-soft X-ray emission, and inverse Compton for the X-ray through the $\gamma$-ray portion of their spectra, with the ultimate source of power provided by the accretion of matter onto a supermassive black hole where the disk is threaded by a magnetic field [5]. There, the initial transport of energy is likely via strong magnetic field, culminating in acceleration of particles to relativistic energies, although the precise location of the conversion of the magnetically- to matter-dominated structure is still in question (see, e.g., [16]).

The flat spectrum radio quasar 3C 279 was the first bright $\gamma$-ray blazar reported by the EGRET instrument aboard the Compton Gamma-Ray Observatory to show strong and rapidly variable $\gamma$-ray emission (17 10, 19]); recently, it also has been detected at photon energies above $100 \mathrm{GeV}$ by the MAGIC ground-based Cherenkov telescope ([3). This blazar, at the redshift $z=0.536$, harbors a black hole with mass $M \simeq(3-8) \times 10^{8} M_{\odot}\left(\right.$ (20]15]); for specificity, we adopt $6 \times 10^{8} M_{\odot}$. It shows superluminal expansion best described as the jet material propagating with the bulk Lorentz factor $\Gamma_{\text {jet }}=16 \pm 3$ at a small angle $\left(\theta \sim 2^{\circ}\right)$ to our line of sight $(\sqrt{9})$. In this paper, we report the results of the first-year Fermi-LAT and multi-band campaign of 3C 279 including optical polarimetric observations. Details of the campaign results and discussions are also described in [1].

\section{Fermi-LAT observations and data reductions}

The successful launch and deployment of the Fermi Gamma-ray Space Telescope inaugurated a new capability to monitor the $\gamma$-ray sky. The main instrument on-board Fermi, the Large Area Telescope (LAT: [4]) features a wide (2.4 steradian) instantaneous field of view and rocks by 35 $50^{\circ}$ north and south of the orbital plane every second orbit, thus observing the entire sky every $\sim 3$ hours and allowing an essentially uninterrupted flux history of 3C 279 in the $\gamma$-ray band. The data used here comprise 11-month observations obtained between August 4, 2008 and June 9, 2009 (54682 - 54991 MJD). The LAT data have been analyzed by using a standard LAT analysis tool (ScienceTools version 9.15.3) with the "diffuse" event selection cut and "P6_V3_DIFFUSE" for the instrumental response function. The events were extracted in the range between $200 \mathrm{MeV}$ and $300 \mathrm{GeV}$ and within a $17^{\circ}$ acceptance cone of the Region of Interest (ROI) centered on the location of $3 \mathrm{C} 279\left(\mathrm{RA}=195.047^{\circ}, \mathrm{DEC}=-5.789^{\circ}, \mathrm{J} 2000\right)$ The fluxes are calculated by unbinned maximum likelihood technique with a source spectral model of a simple power-law $(\mathrm{d} F / \mathrm{d} E=$ $A \cdot\left(E / E_{0}\right)^{-\Gamma}$ ) taking into account the Galactic diffuse emission (model "gll_iem_v02.fit" prepared with GALPROP code [17]), an isotropic component (extragalactic and instrumental backgrounds) and contributions from two nearby bright sources, 3C 273 and 1FGL J1231.1-1410.

\section{Results}

In Figure 1 we plot the flux history in the $\gamma$-ray band by Fermi-LAT, as well as in the X-ray band measured by RXTE-PCA and Swift-XRT, optical, infrared and radio bands by the GASP- 


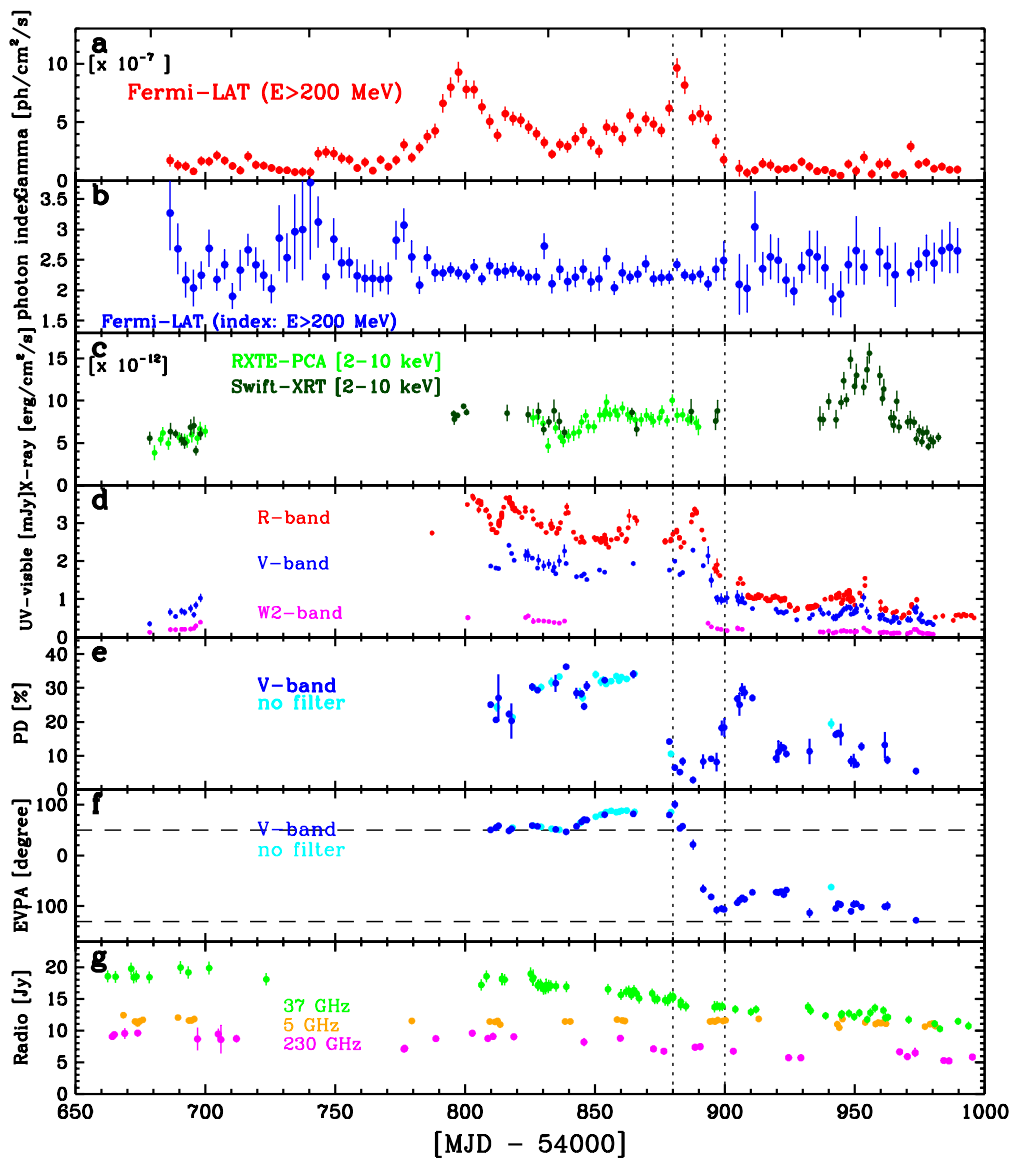

Figure 1: History of fluxes in $\gamma$-ray above $200 \mathrm{MeV}$ (a), X-ray of 2-10 keV (c), optical-UV (d), radio (g) bands as well as $\gamma$-ray photon index (b), optical polarization degree (e) and angle (f) of 3C 279 between July 2008 and June 2009 (54650 and 55000 MJD). The two dashed vertical lines correspond to 54880 and $54900 \mathrm{MJD}$, respectively. The full version of the light curves of the campaign including some additional bands can be seen in [1].

WEBT observatories ${ }^{1}$ and Swift-UVOT. Polarization information in the optical band has been also provided by Kanata (V-band:[18]) and KVA (no filter) telescopes.

Towards the end of the high state there is a sharp $\gamma$-ray flare at 54880 MJD. This sharp $\gamma$-ray flare coincides with a significant drop of the level of optical polarization (polarization degree: PD), from $\sim 30 \%$ down to a few $\%$, lasting for $\Delta t \sim 20$ days. Subsequently, both $\gamma$-ray and optical fluxes gradually decrease together and reach the quiescent level, followed by a temporary recovery of the high degree of polarization. This event is associated with a dramatic change of the electric vector position angle (EVPA) of the polarization, in contrast to being relatively constant before the event at $\sim 50^{\circ}$ (parallel to the jet direction observed by Very Long Baseline Interferometry observations in radio bands; see, e.g., [9]). Since the EVPA has $\pm 180^{\circ} \times n$ ambiguity, we selected values on the assumption of a smooth change of the EVPA, such that it would follow the overall trend. The polarization angle increases slightly at 54880 MJD - coincident with the $\gamma$-ray flare -

\footnotetext{
${ }^{1}$ See details of the program in http://www. to.astro.it/blazars/webt/gasp/homepage.html
} 


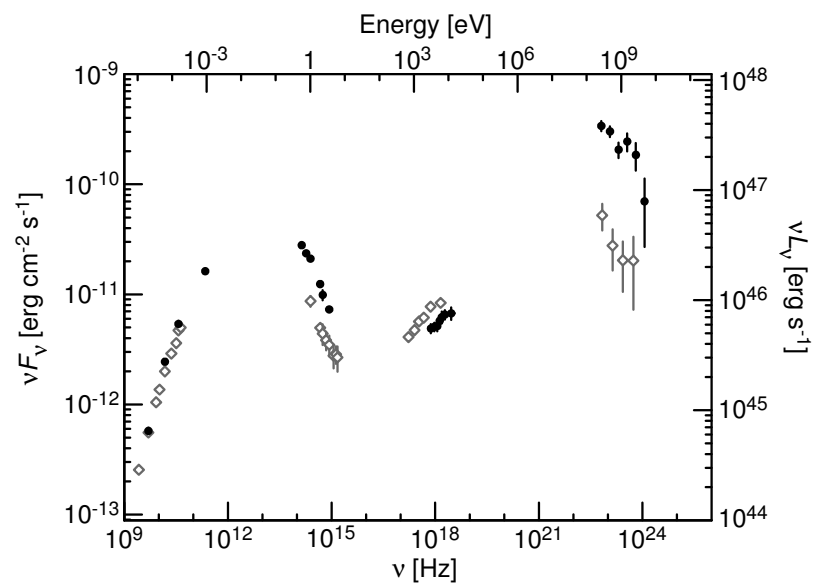

Figure 2: Energy spectrum from radio to $\gamma$-ray band of 3C 279 at two different epochs. The filled circle points were taken between 54880 and $54885 \mathrm{MJD}$, corresponding to the first five days of the sharp $\gamma$-ray flare accompanying the dramatic polarization change event while the open diamond points were taken between 54950 and $54960 \mathrm{MJD}$, around the peak of the isolated X-ray flare. The original version is taken from [1].

then decreases by $208^{\circ}$ with an average rate of $\sim 12^{\circ}$ per day, and returns to a level nearly exactly $180^{\circ}$ from the original level, resembling closely the behavior of optical polarization measured in BL Lacertae ([14]), but at a 4 times slower rate. This clearly indicates that the sharp $\gamma$-ray flare is unambiguously correlated with the dramatic change of optical polarization due to a single, coherent event, rather than a superposition of multiple shorter duration events.

Concurrent X-ray observations indicate relatively steady flux during the $\gamma$-ray flare associated with the optical polarization change, but reveal a significant, symmetrical flare at 54950 MJD with duration of $\sim 20$ days, similar to the duration of the $\gamma$-ray flare. Importantly, this X-ray flare is not accompanied by a prominent optical or $\gamma$-ray flare. The X-ray spectrum during the isolated flare remains much harder than the optical spectrum (see Figure 2),

\section{Discussions}

The gradual rotation of the polarization angle is unlikely to originate in an axially symmetric jet since any compression of the jet plasma by, e.g., a perpendicular shock moving along the jet and viewed at a small but constant angle to the jet axis would change the degree of polarization, but would not result in a gradual change of EVPA. Instead, it can reflect a non-axisymmetric magnetic field distribution (as in, e.g., [11]). The first possibility may be due to propagation of an emission knot following a helical path in a magnetically dominated jet as was recently investigated in the context of the optical polarization event seen in BL Lacertae ([14]) or may involve the "global" bending of a jet. Since the magnetic field in the emission region is anisotropic - presumably concentrated in the plane of a shock / disturbance propagating along the jet - the degree and angle of observed polarization then depends on the instantaneous angle $\theta$ of the direction of motion of the radiating material to the line of sight. The maximum rotation rate of the polarization angle would correspond to the minimum of $\theta$. Similar geometry - albeit on larger scales - has been observed in another blazar, PKS 1510-089 ([8]). Nonetheless, in both scenarios, the coherent polarization event is produced by 
a density pattern co-moving along the jet, and therefore, it is possible to estimate the distance traveled by the emitting material during the flare $\Delta r_{\text {event }}$; this in turn allows us to constrain the distance of the dissipation region from the black hole $r_{\text {event }} \geq \Delta r_{\text {event }} \sim 10^{19}\left(\Delta t_{\text {event }} / 20\right.$ days $)\left(\Gamma_{\text {jet }} / 15\right)^{2} \mathrm{~cm}$, which is $\sim 5$ orders of magnitude larger than the gravitational radius of the black hole in 3C 279 . The constraints on the distance of the dissipation region can be relaxed under "flow-through" scenarios - where the emission patterns may move much slower than the bulk speed of the jet or not propagate at all: one such example is the model involving swings ("wobbling") of the jet associated with jet instabilities such that its boundary moves relative to our line of sight. In this case the time scale for the observed variation is the time scale for the jet motion. Consequently, the emission region easily can be much closer (by a factor $\Gamma_{\text {jet }}^{2}$ ) to the black hole than in the "helical" or "bent jet" scenarios, since the natural radial scale for $\Delta t_{\text {event }} \sim 20$ days is $r_{\text {event }} \sim c \Delta t_{\text {event }} \sim 500-1000$ gravitational radii (see, e.g., [13]).

This leaves us with three viable possibilities. Both the scenario involving a knot propagating along the helical magnetic field lines and the "flow-through" scenario above imply that the rotation of the polarization angle should be preferentially following the same direction, since in those two models the twist presumably originates in the inner accretion disk. In our case, we observe the rotation of the polarization angle that is opposite in direction than that measured previously ([12]), leaving us with the "bent jet" model combined with a small swing of the jet as the most compelling.

The 'isolated' X-ray flare around 54950 MJD with a similar duration of the $\gamma$-ray flare suggests the X-ray photons are produced at a comparable distance from the black hole as the optical $/ \gamma$-ray photons. The hard photon index (harder than 2) cannot be explained by a temporary extension of the high-energy tail of the synchrotron emission, but instead, may be generated by inverse-Compton scattering by low-energy electrons. However, the similarity of profiles of the $\gamma$-ray and X-ray flares argues against the latter being just a delayed version of the former due to, e.g., particle cooling. Therefore, it must be produced independently by another mechanism involving primarily lower energy electrons.

The dominant source of "seed" photons for inverse-Compton scattering depends on the distance of the dissipation event from the central black hole. At the distance of production of the polarization/ $\gamma$-ray flare event in $3 \mathrm{C} 279, r_{\text {event }} \sim 10^{19} \mathrm{~cm}$, the radiation fields from the accretion disk or broad line clouds are not important (ref. [16]); instead, both the synchrotron jet emission and the infrared photons emitted by a warm dust located in the circumnuclear molecular torus are likely to be important.

\section{Concluding remarks}

The superb $\gamma$-ray data provided by the Fermi Gamma-ray Space Telescope, in conjunction with well-sampled data in other bands, is already providing new insight on the structure of relativistic jets associated with massive black holes in centers of galaxies. The close association of the energetically-dominant $\gamma$-ray flare with a smooth, continuous change of the optical polarization angle provides a measure of the spatial scale (both size and location) of the coherent event and implies a bend of the trajectory of the emitting material. The event takes place at few parsecs from the black hole and production of $\gamma$-rays is likely to be dominated by Comptonization of infrared radiation of dust located in the surrounding molecular torus. However, the detection of the isolated 
$\mathrm{X}$-ray flare challenges the simple, one-zone emission models, rendering them too simple. More detailed study of spectral energy distributions including data provided by sensitive observations, for example, by Spitzer for far-to-mid infrared bands, Suzaku and XMM-Newton for soft-to-hard $\mathrm{X}$-ray bands would provide us with useful information to constrain emission models and physical parameters in the jets.

\section{Acknowledgments}

The Fermi-LAT Collaboration acknowledges support from a number of agencies and institutes for both development and the operation of the LAT as well as scientific data analysis. These include NASA and DOE in the United States, CEA/Irfu and IN2P3/CNRS in France, ASI and INFN in Italy, MEXT, KEK, and JAXA in Japan, and the K. A. Wallenberg Foundation, the Swedish Research Council and the National Space Board in Sweden. Additional support from INAF in Italy and CNES in France for science analysis during the operations phase is also gratefully acknowledged.

\section{References}

[1] Abdo, A. A., et al. Nature, 463, 919 (2010)

[2] Abdo, A. A., et al. Astrophys. J., 715, 429 (2010b)

[3] Albert, J., et al. Science, 320, 1752 (2008)

[4] Atwood, W. B., et al. Astrophys. J., 697, 1071 (2009)

[5] Blandford, R., \& Payne, D. MNRAS, 199, 883 (1982)

[6] Dermer, C., Schlickheiser, R., \& Mastichiadis, A. Astron. Astrophys., 256, L27 (1992)

[7] Hartman, R.. C., et al. Astrophys. J. Lett., 385, L1 (1992)

[8] Homan, D. C., et al. Astrophys. J., 580, 742 (2002)

[9] Jorstad, S. G., et al. Astron. J., 130, 1418 (2005)

[10] Kniffen, D. A., et al. Astrophys. J., 411, 133 (1994)

[11] Konigl, A., \& Choudhuri, A. R. Astrophys. J. 289, 188 (1985)

[12] Larionov, V. M., et al. Astron. Astrophys. 492, 389 (2008)

[13] Lyutikov, M., Pariev, V. I., \& Blandford, R. Astrophys. J. 597, 998 (2003)

[14] Marscher, A. P., et al. Nature, 452, 966 (2008)

[15] Nilsson, K., et al. Astron. Astrophys., 505, 601 (2009)

[16] Sikora, M., et al. ApJ, 704, 38 (2009)

[17] Strong, A. W., Moskalenko, I. \& Reimer O. Astrophys. J., 613, 962 (2004)

[18] Watanabe, M., et al. Pub. Astron. Soc. Pacif., 117, 870 (2005)

[19] Wehrle, A. E., et al. Astrophys. J., 497, 178 (1998)

[20] Woo, J.-H., \& Urry, C. M. Astrophys. J., 579, 530 (2002) 